\title{
A Comparison between Therapeutic Effect of Granulocyte Colony-stimulating Factor and Methylprednisolone in Treatment of Patients with Acute Traumatic Spinal Cord Injury
}

\author{
Shahrokh Yousefzadeh-Chabok ${ }^{1}$, Babak Alijani ${ }^{2 *}$, Mohammadreza Emamhadi ${ }^{2}$, Hamid Behzadnia ${ }^{3}$, \\ Siavash Dehghani ${ }^{4}$, Alireza Razzaghi ${ }^{5}$, Shabnam Golmohammadi 4
}

\begin{abstract}
${ }^{1}$ MD, Professor of Neurosurgery, Guilan Road Trauma Research Center, Guilan University of Medical Sciences, Rasht, Guilan, Iran
${ }^{2} \mathrm{MD}$, Associate Professor of Neurosurgery,Guilan Road Trauma Research Center, Guilan University of Medical Sciences, Rasht, Guilan, Iran

${ }^{3}$ MD, Assistant Professor of Neurosurgery, Guilan Road Trauma Research Center, Guilan University of Medical Sciences, Rasht, Guilan, Iran

${ }^{4} \mathrm{MD}$, Senior Resident of Neurosurgery, Poursina Hospital, Guilan University of Medical Sciences, Rasht, Guilan, Iran

${ }^{5} \mathrm{MSc}$ in Epidemiology, Guilan Road Trauma Research Center, Poursina Hospital, Guilan University of Medical Sciences, Rasht, Guilan, Iran

* Corresponding Author Address: Poursina Hospital, Guilan University of Medical Sciences, Rasht, Guilan, Iran. Tel:+981333368773, Fax: +981333339842. E-mail: babakalij@gmail.com
\end{abstract}

Article Type: Research Article

Received: March 1, 2016, Last Revised: May 16, 2016, Accepted: May 30, 2016

\section{Abstract}

Background \& Aim: Spinal cord injury (SCI) is one of the worst kinds of traumatic injuries with remarkable social and economic effects on communities.

Methods \& Materials/Patients: In this prospective randomized clinical trial, 122 patients with traumatic spinal cord injury were admitted to Poursina hospital within 48 hours of injury to compare granulocyte colony stimulating factor (G-CSF) and high-dose methylprednisolone as neuroprotective therapy.

Results: In this research, 122 patients were studied out of whom 62 patients were included in the granulociote colony-stimulating factor (G-CSF) group with a mean age of 40.4, and 60 patients in the methylprednisolone group with an average age of 40.10 years. 55 out of 122 patients (45\%) were completely paralyzed (Grade AIS:A) and $67(55 \%)$ were with incomplete spinal injury (Grade AIS:B,C,D). The average American spinal injury association (ASIA) sensory scores in the two groups were similarly compared in the same four time intervals, and $\mathrm{p}$ values were $0.7,0.3,0.2$, and 0.1 . They were not statistically significant.

Conclusion: According to the results, the G-CSF was shown to be beneficial in average ASIA motor and sensory scores in the two groups of male patients at six months of onset of G-CSF treatment ( $\mathrm{p}$ value $=0.04$ ), and average ASIA motor scores improvement in the falls subgroup patients also differed significantly within six months of onset of G-CSF treatment ( $p$ value $=0.03$ ). A multicentre prospective randomized clinical trial to compare the placebo effect with G-CSF protocol's treatment, and also assessment of the cost benefits of the common medical treatment versus G-CSF are needed.

Keywords: Granulocyte; Colony-stimulating; Methylprednisolone; Treatment

Please cite this paper as: Yousefzadeh-Chabok Sh, Alijani B, Emamhadi M, Behzadnia H, Dehghani S, Razzaghi A, Golmohammadi Sh A Comparison between Therapeutic Effect of Granulocyte Colony-stimulating Factor and Methylprednisolone in Treatment of Patients with Acute Traumatic Spinal Cord Injury. Iran. J. Neurosurg. 2016;2(1):11-14

\section{Introduction}

Spinal cord injury (SCI) is one of the worst kinds of traumatic injuries with great social and economic impacts on communities. An estimated 5.2 million people are living with spinal cord injuries with incidence of 130,000 new cases per year all over the world (1). The estimated annual cost of care for patients with SCI in the United States is 73.9 billion (2).

During the acute phase of SCI, the focal mechanical impact triggers a cascade of destructive processes in which multiple secondary damages such as necrosis and apoptotic death of neurons and irreversible axonal damage and demyelization play role $(3,4)$. Spinal cord injury causes rapid loss of axons that occurs within a week of the injury. Although most of this loss occurs during the first week but axons remodeling, tissue remodeling, and pathology can be detected up to 10 weeks after injury (5). Effective treatment for spinal cord injuries is limited by complex pathophysiology of causative mechanisms (6). There are limited medical therapies for spinal cord injury. According to a study of the national acute spinal cord injury study 2 (NASCIS-2), the use of high-dose methylprednisolone has been confirmed as the standard treatment for spinal cord injuries. Studies have shown that methylphenidate prednisolone used within eight hours of injury have improved sensory and motor outcomes (7, 8). However, several studies have shown that after this treatment, side effects, respiratory system and digestive organs damage occurred frequently and were often serious (9-14).

Granulocyte colony-stimulating factor (GCS-F) is a glycoprotein which is proposed to have anti-inflammatory effects (15). Numerous reports show that G-CSF has antiinflammatory effects and inhibits apoptotic pathways (1621). G-CSF receptors are expressed in neural stem cells and cause neuronal differentiation in vitro. It protects neural tissue from glutamate-induced neurotoxicity 
during spinal cord injury process (22) .

Based on these results, we performed a clinical trial and compared the safety and feasibility of G-CSF and high-dose methylprednisolone as neuroprotective therapy in patients with acute SCI.

\section{Methods and Materials/Patients}

The study was designed as a prospective randomized clinical trial to compare G-CSF and high-dose methylprednisolone as neuroprotective therapy in patients with acute traumatic spinal cord injury.

122 patients with traumatic spinal cord injury were admitted to Poursina hospital within 48 hours of injury. They were enrolled into the study according to the following inclusion criteria:

- Acute (up to 48 hours) traumatic spinal cord injury

- Age between 18 to 65 years old

- Absence of intracranial lesion such as a tumor, infection, ischemia or trauma.

- No past history of major bleeding requiring blood transfusion

- No past history of leukopenia or thrombocytopenia

- No past history of hepatic or renal dysfunction

- No past history of severe heart failure

- Absence of splenomegaly or a related history

- No evidence of malignant disease in the past five years

- No pregnancy or lactation in patients

Patients with history of major bleeding, blood dyscrasia, renal and hepatic failure and history of malignant disease in the past five years were excluded. Written informed consent was obtained from all patients prior to the treatment in both groups.

The baseline grade of spinal cord injury using AIS scoring system and sensory and motor function score of patients were measured using the ASIA scoring system.

The patients were randomized into two groups. In the first group, treatment with bolus dose of $15 \mathrm{mg} / \mathrm{kg}$ of methylprednisolone sodium succinate during the first eight hours after injury was begun. If the treatment was started within three hours after injury, methylprednisolone infusion continued with a dose of $5.4 \mathrm{mg}$ / $\mathrm{kg} / \mathrm{h}$ to 23 hours later on. If the treatment was started within three to eight hours of injury onset, infusion of methylprednisolone continued up to 47 hours later on with same dosages.

In the second group, treatment with G-CSF was administered at a dose of 10 micrograms $/ \mathrm{kg}$ daily for five consecutive days. Vital signs, clinical condition and blood profile were assessed for seven days according to the manufacturer's protocol. The patients were checked for possible side effects. In case of pain or fever over 38 ${ }^{\circ} \mathrm{C}$ the patients were given nonsteroidal anti-inflammatory drugs such as diclofenac. Treatment was ended in the case of severe side effects.

In both groups of patients, recovery of sensory and motor function was assessed by ASIA scoring system and AIS grading system one week, three months and six months after the intervention consecutively.

\section{Results}

In current research, 122 patients were studied. Out of 62 patients in the G-CSF group with a mean age of $40.4(\mathrm{SD}=16.02)$, 47 patients $(75.8 \%)$ were male and $15(24.2 \%)$ female. The methylprednisolone group also included 60 patients with an average age of 40.10 years $(\mathrm{SD}=13.22)$, in which 48 patients $(80 \%)$ were male and $12(20 \%)$ female.

$55(45 \%)$ out of 122 patients were completely paralyzed (Grade AIS: A) and 67 (55\%) were with incomplete spinal injury (Grade AIS: B, C, D).

The most common cause of trauma was motor car accident with 71 cases $(58.2 \%)$ followed by falls with 43 cases $(35.2 \%)$, conflicts with 5 cases $(4.1 \%)$ and sports injury with 3 cases $(2.5 \%)$. Based on the level of damage, most of them were cervical injuries with 80 cases $(65.6 \%)$.

The average ASIA motor scores in the two groups in sequential intervals of first day, seventh day, three and six months later were compared in two groups. The p-values were $0.7,0.4,0.1$ and 0.05 , respectively. The average ASIA sensory scores in the two groups were similarly compared in the same time intervals and $\mathrm{p}$ values were $0.7,0.3,0.2$, and 0.1 , respectively. They were not shown to be statistically significant. Only the comparison between average ASIA motor and sensory scores in the two groups of male patients sub-group differed significantly within six months of onset of G-CSF treatment ( $p$-value $=0.04)$. The average ASIA motor scores improvement in the falls sub-group patients also differed significantly within six months of onset of G-CSF treatment ( $p$-value $=0.03$ ).

The difference observed in the prevalence of AIS grades at the time of acute traumatic SCI examined in patients treated with G-CSF compared to those treated with methylprednisolone was not statistically significant in any of the mentioned time intervals $(\mathrm{p}>0.05)$.

\section{Conclusion}

122 patients with traumatic SCI admitted to Poursina hospital within 48 hours of injury were assessed by prospective randomized clinical trial comparing G-CSF and high-dose methylprednisolone as neuroprotective therapy. According to the results, there was no significant difference between two groups in terms of age, sex and cause of trauma.

In the present study, neurological improvement between the two groups of G-CSF and high-dose methylprednisolone based on average ASIA motor and sensory scores and AIS grading scales in the two groups in sequential intervals of the first day and seventh day, three and six months after the intervention were compared. There was a significant difference between the two groups in the average ASIA motor and sensory scores after six months of injury in male patients ( $p$-value $=0.04$ ), as well as in patients injured by falls ( $p$-value $=0.03)$. However, overall improvement of ASIA motor, sensory scores and AIS grading scales did not differ significantly (p-value $>0.05$ ).

In review of similar articles, Takahashi and colleagues in 2012 compared two different G-CSF treatment's protocols with five and 10 micrograms $/ \mathrm{kg} /$ day of IV infusion for five days consequently in patients at first 24 hours of acute spinal injuries to show efficacy and safety of G-CSF treatment. It showed progression in neurological outcomes, but the samples were few to be fully comprehended. It also only analyzed the partial spinal cord injured patients (23).

Saberi and colleagues in 2012 compared two partial and complete chronic spinal injury groups of patients treated by five micrograms/kg/day subcutaneous injection for seven days consecutively. Patients with incomplete SCI had greater improvement in motor and grading ASIA scores compared to complete SCI patients. The higher grades of injuries were correlated with poorer results. The article only assessed the chronic phase of injury and did not compare it with other treatment modalities (24).

In a retrospective study conducted by Kamiya and colleagues in 2015, two groups of nonrandomized partial SCI treated by G-CSF and high-dose methylprednisolone followed up to three months were compared. It showed superior improvement in motor and grading ASIA score in G-CSF group. It lacked long-term follow-up in non-randomized trial and also did not 
include complete SCI patients in the assessment (25).

Inada and colleagues in 2014 also compared patients with traumatic spinal cord injury admitted within 48 hours of injury treated by G-CSF and compared it with control group without medical neuroprotective treatment in non-randomized prospective trial and followed patients for one year later on, in seven days, three months, six months and one year after injury. It showed improvement in motor and grading ASIA score in G-CSF treated ones compared to control group at first week and a year later, though it lacked randomization and the G-CSF intervention was not compared to other modalities (26).

In our study, the sampling was greater in number than that in all other similar articles, and it is the only prospective randomized clinical trial that compared G-CSF and high-dose methylprednisolone as neuroprotective therapy in patients with acute traumatic spinal cord injury. Moreover, it compared all complete and partial SCI cases (grades A, B, C and D).

\section{Recommendation}

We recommend the multicenter prospective randomized clinical trial to compare the placebo effect with that of G-CSF protocol's treatment, and also assessment of the cost benefits of the common medical treatment with those of G-CSF.

\section{Funding}

None.

\section{Conflicts of Interest}

\section{The authors have no conflicts of interest.}

\section{References}

1. Thuret S, Moon LD, Gage FH. Therapeutic interventions after spinal cord injury. Nature reviews Neuroscience. 2006;7(8):628-43.

2. Weaver FM, Hammond MC, Guihan M, Hendricks RD. Department of Veterans Affairs Quality Enhancement Research Initiative for spinal cord injury. Medical care. 2000;38(6 Suppl 1):I82-91.

3. Rowland JW, Hawryluk GW, Kwon B, Fehlings MG. Current status of acute spinal cord injury pathophysiology and emerging therapies: promise on the horizon. Neurosurgical Focus. 2008;25(5):E2.

4. Mueller CA, Schluesener HJ, Conrad S, Pietsch T, Schwab JM. Spinal cord injury-induced expression of the immune-regulatory chemokine interleukin-16 caused by activated microglia/macrophages and CD8+ cells. Journal of Neurosurgery Spine. 2006;4(3):233-40.

5. Ek CJ, Habgood MD, Dennis R, Dziegielewska KM, Mallard C, Wheaton B, et al. Pathological changes in the white matter after spinal contusion injury in the rat. PloS one. 2012;7(8):e43484.

6. Norenberg MD, Smith J, Marcillo A. The pathology of human spinal cord injury: defining the problems. Journal of Neurotrauma. 2004;21(4):429-40.

7. Bracken MB, Shepard MJ, Collins WF, Holford TR, Young W, Baskin DS, et al. A randomized, controlled trial of methylprednisolone or naloxone in the treatment of acute spinal-cord injury. Results of the Second National Acute Spinal Cord Injury Study. The New England Journal of Medicine. 1990;322(20):140511.

8. Bracken MB, Shepard MJ, Holford TR, Leo-Summers L, Aldrich EF, Fazl $\mathrm{M}$, et al. Administration of methylprednisolone for 24 or 48 hours or tirilazad mesylate for 48 hours in the treatment of acute spinal cord injury. Results of the Third National Acute Spinal Cord Injury Randomized Controlled Trial. National Acute Spinal Cord Injury Study. Jama. 1997;277(20):1597-604.

9. Hugenholtz H, Cass DE, Dvorak MF, Fewer DH, Fox RJ, Izukawa DM, et al. High-dose methylprednisolone for acute closed spinal cord injury--only a treatment option. The Canadian journal of neurological sciences Le Journal Canadien des Sciences Neurologiques. 2002;29(3):227-35.

10. Hurlbert RJ. The role of steroids in acute spinal cord injury: an evidencebased analysis. Spine. 2001;26(24 Suppl):S39-46.

11. Ito Y, Sugimoto Y, Tomioka M, Kai N, Tanaka M. Does high dose methylprednisolone sodium succinate really improve neurological status in patient with acute cervical cord injury?: a prospective study about neurological recovery and early complications. Spine. 2009;34(20):2121-4

12. Matsumoto T, Tamaki T, Kawakami M, Yoshida M, Ando M, Yamada H. Early complications of high-dose methylprednisolone sodium succinate treatment in the follow-up of acute cervical spinal cord injury. Spine. 2001;26(4):426-30. 13. Pollard ME, Apple DF. Factors associated with improved neurologic outcomes in patients with incomplete tetraplegia. Spine. 2003;28(1):33-9.

14. Rabchevsky AG, Fugaccia I, Sullivan PG, Blades DA, Scheff SW. Efficacy of methylprednisolone therapy for the injured rat spinal cord. Journal of Neuroscience Research. 2002;68(1):7-18.

15. Boneberg EM, Hartung T. Molecular aspects of anti-inflammatory action of G-CSF. Inflammation research : official journal of the European Histamine Research Society [et al]. 2002;51(3):119-28.

16. Ha Y, Kim YS, Cho JM, Yoon SH, Park SR, Yoon DH, et al. Role of granulocyte-macrophage colony-stimulating factor in preventing apoptosis and improving functional outcome in experimental spinal cord contusion injury. Journal of Neurosurgery Spine. 2005;2(1):55-61

17. Huang HY, Lin SZ, Kuo JS, Chen WF, Wang MJ. G-CSF protects dopaminergic neurons from 6-OHDA-induced toxicity via the ERK pathway. Neurobiology of Aging. 2007;28(8):1258-69.

18. Chen WF, Jean YH, Sung CS, Wu GJ, Huang SY, Ho JT, et al. Intrathecally injected granulocyte colony-stimulating factor produced neuroprotective effects in spinal cord ischemia via the mitogen-activated protein kinase and Akt pathways. Neuroscience. 2008;153(1):31-43.

19. Lu CZ, Xiao BG. Neuroprotection of G-CSF in cerebral ischemia. Frontiers in Bioscience: a Journal and Virtual Library. 2007;12:2869-75.

20. Sehara Y, Hayashi T, Deguchi K, Zhang H, Tsuchiya A, Yamashita T, et al. Decreased focal inflammatory response by G-CSF may improve stroke outcome after transient middle cerebral artery occlusion in rats. Journal of Neuroscience Research. 2007;85(10):2167-74

21. Schneider A, Kruger C, Steigleder T, Weber D, Pitzer C, Laage R, et al. The hematopoietic factor G-CSF is a neuronal ligand that counteracts programmed cell death and drives neurogenesis. The Journal of Clinical Investigation. 2005;115(8):2083-98.

22. Schabitz WR, Kollmar R, Schwaninger M, Juettler E, Bardutzky J, Scholzke MN, et al. Neuroprotective effect of granulocyte colony-stimulating factor after focal cerebral ischemia. Stroke; a Journal of Cerebral Circulation. 2003;34(3):745-51.

23. Takahashi H, Yamazaki M, Okawa A, Sakuma T, Kato K, Hashimoto M, et al. Neuroprotective therapy using granulocyte colony-stimulating factor for acute spinal cord injury: a phase I/IIa clinical trial. European Spine Journal : official publication of the European Spine Society, the European Spinal Deformity Society, and the European Section of the Cervical Spine Research Society. 2012;21(12):2580-7.

24. Derakhshanrad N, Saberi H, Yekaninejad MS, Eskandari G, Mardani A, Rahdari F, et al. Safety of granulocyte colony-stimulating factor (G-CSF) administration for postrehabilitated motor complete spinal cord injury patients: an open-label, phase I study. Cell Transplantation. 2013;221:S139-46.

25. Kamiya K, Koda M, Furuya T, Kato K, Takahashi H, Sakuma T, et al. Neuroprotective therapy with granulocyte colony-stimulating factor in acute spinal cord injury: a comparison with high-dose methylprednisolone as a historical control. European Spine Journal: Official Publication of the European Spine Society, the European Spinal Deformity Society, and the European Section of the Cervical Spine Research Society. 2015;24(5):963-7.

26. Inada T, Takahashi H, Yamazaki M, Okawa A, Sakuma T, Kato K, et al. Multicenter prospective nonrandomized controlled clinical trial to prove neurotherapeutic effects of granulocyte colony-stimulating factor for acute spinal cord injury: analyses of follow-up cases after at least 1 year. Spine. 2014;39(3):213-9.

\section{Comments}

Reading with interest the manuscript 'A Comparison between Therapeutic Effect of Granulocyte Colony-stimulating Factor and Methylprednisolone in Treatment of Patients with Acute Traumatic Spinal Cord Injury (SCI)' the authors have tried to conduct an RCT including 122 cases of SCI treated by two different medications and demonstrated that the results of short and intermediate outcomes did not differ in a significant manner. Certainly the results are reportable as the preliminary report but I would like to add a commentary about such kind of studies till the other researchers might include the notifications;

1. The inclusion criteria should be very strict in any RCT, especially for SCI. So, including cases with different levels of AIS scoring and not sub classifying them in the tables and rows for analysis, make the outputs a kind of 'mix-up'. 
2. An associated file or link should be attached for the readers to see, the characteristics of the observers' examining the cases in the emergency departments, the neuroradiologists, the neurologists, the attending surgeons, the types of rehabilitations for each case, the follow up images and so on.

3. Comparing two medications, both of them needed to be administered before 12-24 hours after the event looks impossible in the community with patient transfer time longer than 24 hours on average!!

4. Considering this pre-requisite, the bias of selection would be the major hazard for randomization.

5. The introduction looks very long and those statements denoting the pharmaceutical characteristics of each medicament can be transferred to discussion.

6. It would be better to quote only similar references reporting only the targeted interventions rather general discussion about such kind of interventions.

Abbas Amirjamshidi, MD, MPH, Professor of Neurosurgery, Sina Hospital, Tehran University of Medical Sciences (TUMS) 